\title{
COMPUESTO DE HIDROXIAPATITA-LIGNINA COMO ÓSEO-INTEGRADOR ENTRE TEJIDO ÓSEO E IMPLANTE METÁLICO: EVALUACIÓN MECÁNICA
}

\section{HYDROXYAPATITE-LIGNIN COMPOUND AS AN OSSEUS-INTEGRATOR BETWEEN BONE TISSUE AND A METALLIC IMPLANT: MECHANICAL EVALUATION}

\author{
Mastoby Martínez ${ }^{1}$, Andrea Pacheco², Ricardo Della ${ }^{3}$, Emily Correna ${ }^{4}$, \\ Liana Mesquita ${ }^{5}$, Glaucia Oliveira ${ }^{6}$, Sheila Bicalho ${ }^{7}$
}

${ }^{1}$ M.Sc, Profesor. Universidad de Córdoba. A.A. 354 Montería, Córdoba-Colombia, e-mail: mastobymvz@hotmail.com, ${ }^{2}$ Ph.D, Profesor Universidad Federal de Viçosa. CEP 36570-000, Viçosa-MG-Brasil. rdlucia@ufv.br, ${ }^{3}$ Ph.D, Profesor Universidad Federal de Viçosa. CEP 36570-000, Viçosa-MG-Brasil. rdlucia@ufv.br, ${ }^{4}$ M.Sc, Doctorando - Universidad Federal de Viçosa. CEP 36570-000 Viçosa-MG-Brasil, emiliccarlo@hotmail.com, ${ }^{5}$ M.Sc, Doctorando - Universidad Federal de Viçosa. CEP 36570-000 Viçosa-MG-Brasil. lilymesquita@gmail.com, ${ }^{6}$ Estudiante de Graduación - Universidad Federal de Viçosa, CEP 36570-000 Viçosa-MG-Brasil, glauciamorato@yahoo.com.br, ${ }^{7}$ Sheila Bicalho, Ph.D, Gerente del Laboratorio Químico Ltda. - Belo Horizonte, MG - Brasil, jhs@.org.br.

Rev. U.D.C.A Act. E Div. Cient. 12 (2): 19-26, 2009

\section{RESUMEN}

En el estudio, se evaluó al compuesto de hidroxiapatitalignina como óseo-integrador entre tejido óseo e implante metálico por prueba de tracción, considerándose oportuna la investigación por la necesidad de hallar un biomaterial que guíe o posea propiedades químicas y mecánicas semejantes al hueso, para que reemplace al metilmetacrilato utilizado en la fijación del vástago de prótesis articulares. Se utilizaron 20 conejos Nueva Zelanda; en cada uno de ellos, se trató la tibia izquierda con el compuesto y la tibia derecha sirvió como control. Se realizó una falla ósea de $4 \mathrm{~mm}$ de diámetro en la superficie lateral proximal tibial, hasta alcanzar el canal medular. Del compuesto, 1000mg fueron ablandados con diez gotas de $\mathrm{NaCl}(0,9 \%)$, utilizando parte de la masa para revestir la rosca del clavo intramedular de Schanz en acero de diámetro $2,5 \mathrm{~mm}$ para hueso cortical y, la otra parte, introducida en el canal medular del grupo tratado con ayuda de catéter calibre 16 y jeringa; después, se introdujeron $7 \mathrm{~cm}$ del clavo en el canal medular. Los planos anatómicos incididos se suturaron. Lo mismo se realizó en el grupo control, sin emplear el compuesto. Las evaluaciones, se hicieron a los 8, 30, 60, 90 y 120 días pos cirugía. En la prueba de tracción no se observaron diferencias estadísticas entre grupo tratado y control, a pesar que en el primero el resultado fue el doble de la resistencia del segundo. Basado en los resultados, se concluye que el compuesto de hidroxiapatita-lignina facilita la óseointegración del clavo intramedular.

Palabras clave: Conejo, hueso, bioimplante, regeneración, conducción.

\section{SUMMARY}

This study evaluated the compound hydroxyapatitelignin as an osseous-integrator between bone tissue and a metal implant, using a traction test. The research was considered opportune, due to the necessity to discover a biomaterial, which guides and has chemical and mechanical properties similar to the bone, in order to replace the methyl methacrylate used in the stem 
joint fixation of prosthesis. 20 New Zealand rabbits were employed; in all of them the left tibia was treated with the compound and, the not treated right tibia was considered the control. A bone fail, $4 \mathrm{~mm}$ in diameter, was performed in the proximal lateral tibial surface, up to the marrow channel. $1000 \mathrm{mg}$ of the compound were softened with ten drops of $\mathrm{NaCl}(0.9 \%)$ using part of the dough used to coat the thread of the intra-marrow Schanz steel nail for cortical bone, diameter $2.5 \mathrm{~mm}$. The other part was entered into the spinal channel of the treated group, with the help of a caliber 16-gauge catheter and a syringe. Afterwards, $7 \mathrm{~cm}$ of the nail were introduced into the marrow channel. The affected anatomic parts were sutured. The same was executed with the control group, without the use of the compound. The assessments were performed 8, 30, 60, 90 and 120 days post surgery. In the traction test no statistical differences between treated and control group were observed, despite that in the first group, the result was double the resistance strength than that of the control. Based on the results it is concluded that the lignin-hydroxyapatite compound facilitates the osseous-integration of the intra-marrow nail.

Key words: Rabbit, bone, bio-implant, regeneration, conduction.

\section{INTRODUCCIÓN}

Teniendo en cuenta la propiedad óseo-conductora de la hidroxiapatita y frente a los efectos adversos que tiene la resina acrílica sobre el tejido óseo a la hora de la fijación de prótesis articulares en la ortopedia veterinaria y humana, un grupo de investigadores de la UFV-Brasil, en trabajo conjunto con investigadores del sector privado, se dispusieron en la tarea de conseguir un biomaterial que pudiera guiar el proceso de regeneración ósea hasta el canal medular y que favoreciera la unión biológica del clavo intramedular en este local.

Los substitutos óseos deben ser inertes, degradables o absorbidos, además de favorecer el crecimiento óseo por conducción y, si es posible, por inducción. Estas características dependen de las propiedades físicas y químicas del biomaterial, que deben ser compatibles con las reacciones fisiológicas del hueso (Ayers et al. 1998; Borges et al. 2000).

Los implantes metálicos vienen siendo empleados con dos propósitos desde hace unas décadas: fabricación de prótesis para reemplazar una parte del cuerpo (articulaciones, placas craneales, clavos, entre otras partes) o implantes para la estabilización y la ayuda al proceso normal de reparación de un tejido (por ejemplo, unión de huesos rotos). Entre los metales más utilizados, con estos fines, cabe destacar diferentes clases de aceros inoxidables, aleaciones tales como Co-Cr, CoCr-Mo, Co-Cr-Ni, así como titanio y distintas aleaciones a base de titanio, de aluminio y de vanadio (Singh $\mathcal{E}$ Dahotre, 2007). Los implantes metálicos pueden sufrir un proceso de corrosión, liberando productos al medio y produciendo, consecuentemente, una determinada reacción tisular. Por tanto, la resistencia a la corrosión es quizás el primer requerimiento que debe cumplir un implante de esta naturaleza. Se utilizan metales y aleaciones resistentes a la corrosión, que se consigue por formación de óxidos o películas sólidas de hidróxidos sobre su superficie (Singh E Dahotre, 2007). Por ejemplo, la gran estabilidad química del acero inoxidable, que contiene un mínimo de $10-11 \%$ de cromo, se atribuye a la película de óxido de cromo, presente sobre su superficie, evitando así la corrosión del hierro. Tales películas, se encuentran en estado de equilibrio dinámico con el oxígeno en el entorno local del implante (Lizarbe, 2007; Singh \& Dahotre, 2007).

La corrosión también puede afectar directamente al tejido circundante por alteración directa del entorno químico: cambios electroquímicos que afecten al comportamiento o la conducta celular, liberación de iones metálicos que pueden afectar el metabolismo celular e inducción de una reacción de inflamación crónica por liberación de los productos de corrosión. En segundo lugar, aunque no es frecuente, la fractura de los implantes metálicos podría producir daños más severos que los acaecidos, como consecuencia de la corrosión. Las principales causas de fractura de un biomaterial son el diseño inadecuado del implante (o defectos de manufacturación) y la mala utilización del mismo (por ejemplo, exceso de peso del paciente, excesivo movimiento, entre otros). El término "fatiga", se aplica a las fracturas que surgen como consecuencia de un exceso de carga sobre el implante y que no se producirían si éste se encontrase en una situación estática (Lizarbe, 2007; Singh E Dahotre 2007).

Desde hace tres décadas, uno de los biomateriales más investigados es la hidroxiapatita, que puede ser obtenida de diferentes formas: a partir de corales marinos, del 
propio hueso o sintetizada en laboratorio (Bostrom, 1998; Fehlberg, 2001; Franco et al. 2001).

La hidroxiapatita ya fue utilizada en la corrección de defectos óseos en el hombre y en varias especies animales, con resultados satisfactorios, lo suficiente para ser indicada como alternativa para la injerta ósea en la rutina clínica (Hemmerlè et al. 1997; Legeros 2002; Miranda et al. 2006; Monchau et al. 2002).

La estructura porosa de la hidroxiapatita funciona como soporte pasivo para la neoformación vascular que llevan factores inductores de aposición ósea (Borges et al. 2000). La superficie porosa de la hidroxiapatita parece proveer un substrato adicional a la proliferación del tejido óseo, favoreciendo la unión, la proliferación, la migración y la expresión fenotípica de células óseas, resultando en formación de nuevo hueso, en aposición directa al biomaterial (Moroni et al. 1996; Moroni et al. 1998 a y b; Legeros 2002; Monchau et al. 2002).

El objetivo del presente trabajo fue evaluar a la hidroxiapatita asociada a lignina (HAP-91- L ${ }^{\circledR} \mathrm{JHS}$, Laboratório Químico, Sabará, Brasil) en el proceso de integración entre un implante metálico y el tejido óseo en tibia de conejos adultos de la raza Nueva Zelanda.

\section{MATERIALES Y MÉTODOS}

Esta investigación de tipo cuantitativo fue aprobada por la comisión de Ética del Departamento de Veterinaria de la Universidad Federal de Viçosa - Brasil (proceso $\left.n^{\circ} 58 / 2007\right)$.

Animales. Fueron utilizados 20 conejos machos de la raza Nueva Zelanda, esqueléticamente maduros a la confirmación radiográfica, con edad entre diez y doce meses y peso promedio de cuatro $\mathrm{kg}$ y medio, oriundos del bioterio del Departamento de Veterinaria de la UFV. Tuvieron dos meses para aclimatación al nuevo ambiente, en jaulas individuales, donde recibieron una dosis única de ivermectina $1 \%(0,2 \mathrm{~mL})$, por la vía subcutánea, alimento dos veces al día y agua a voluntad.

Compuesto. La hidroxiapatita sintética (HAP-91 ${ }^{\circledR}$ ) fue producida por precipitación, después de adicionar gota a gota $\mathrm{H}_{3} \mathrm{PO}_{4}$ sobre el $\mathrm{Ca}(\mathrm{OH})_{2}$, obtenido a partir de la calcinación de la calcita y su posterior hidratación por agitación constante. El precipitado fue calcinado a $900^{\circ} \mathrm{C}$. El Ca de la HAP-91 fue determinado por volumetría y espectroscopia de UV-V (1). Después de este proceso, de acuerdo con el fabricante, fue adicionado a la HAP-91 1\% de lignina, derivada del eucalipto, para formar el compuesto de HAP-91 - L. Con este material, se obtuvieron bloques circulares de $4,5 \mathrm{~mm}$ de diámetro, con peso de 200mg, con proporción de $99 \%$ de HAP-91 y $1 \%$ de lignina, que después fueron esterilizados en óxido de etileno a $10 \%$ y embalados en material plástico.

Procedimiento quirúrgico. En el preoperatorio, los animales recibieron $40.0004 \mathrm{UI} / \mathrm{kg}$ de penicilina sódica y procainica, por vía intramuscular, 30 minutos antes de la inducción anestésica. Después fueron sedados con levomepromazina en dosis de $2 \mathrm{mg} / \mathrm{kg}$ y anestesiados con tiletamina/ zolazepam, en dosis de $20 \mathrm{mg} / \mathrm{kg}$, ambos por vía intramuscular. El tercio proximal del fémur hasta el tercio distal de la tibia fueron depilados y la grasa cutánea fue removida con éter.

La anestesia disociativa se complemento con la técnica epidural lumbo-sacra, empleando lidocaína al $2 \%$, en asocio con epinefrina (12,5mcg de epinefrina $+200 \mathrm{mg}$ de lidocaína/mL), a dosis de $1 \mathrm{~mL} / 4 \mathrm{~kg}$. En seguida, los animales fueron posicionados en decúbito lateral izquierdo. El campo operatorio fue embrocado con alcohol iodado y aislado con venda estéril y delimitado con paños de campo apropiados. El abordaje quirúrgico fue basado en la técnica descrita por Piermattei et al. (2006) para perros y gatos y adaptada para conejos, que constó de los siguientes tiempos:

1. Incisión cutánea parapatelar lateral del tercio distal del fémur hasta la diáfisis proximal de la tibia.

2. Incisión del tejido subcutáneo y fascia lata en la misma línea que la incisión cutánea.

3. Desbridamiento de la musculatura cráneo-lateral de la tibia y separación caudal de la misma.

4. Incisión del periostio en la superficie lateral de la porción proximal de la tibia extendiéndose hasta el final de la cresta tibial.

Posteriormente fue realizada en todos los animales un defecto circular en la parte central de la superficie lateral proximal de la tibia, a $2 \mathrm{~cm}$ de la meseta tibial, de aproximadamente $4 \mathrm{~mm}$ de diámetro y profundad suficiente para alcanzar el canal medular, con el auxilio de un taladro manual y broca de igual diámetro. 
En un mismo animal, se consideró la tibia izquierda como experimental y la tibia derecha como control.

En el grupo tratado fue introducido por el defecto cortical un clavo intramedular de Schanz en acero de calibre $1 \mathrm{~mm}$, con el fin de desprender la médula ósea para facilitar su retirada, como también para irritar el endostio por medio de movimientos de fricción repetitivos. Después, para favorecer la retirada de la médula, se inyectó $10 \mathrm{~cm}$ de aire dentro del canal medular con el auxilio de una jeringa desechable y un catéter venular calibre 16 , retirando la mayor cantidad posible.

En un recipiente, cinco comprimidos del compuesto de HAP-91-L ${ }^{\circledR}(1000 \mathrm{mg})$ fueron ablandados con solución fisiológica $0,9 \%$, en una relación de dos gotas por comprimido, resultando una masa que se utilizó para revestir la rosca del clavo intramedular de Schanz en acero de diámetro $2,5 \mathrm{~mm}$, donde la cantidad se determinó como aquella que se necesitó para que la rosca no fuera visible, dejándola secar por diez minutos al ambiente. Al resto de la masa, se le adicionó solución fisiológica a $0,9 \%$, en volumen suficiente para completar los $2 \mathrm{~mL}$, para ser introducida en el canal medular por medio de jeringa desechable y catéter calibre 16. La longitud del clavo fue medida por medio de la radiografía previa de la tibia, desde el local determinado para el defecto hasta la epífisis distal tibial, determinándose un largo de $7 \mathrm{~cm}$ para todos los animales. Después de cortado, el clavo fue introducido por el defecto cortical y su calibre fue suficiente para ocupar aproximadamente 90\% de la cavidad medular. Los tejidos incididos fueron suturados con naylon.

El grupo control pasó por los mismos procedimientos que el tratado, con la excepción de la introducción del compuesto de HAP-91-L ${ }^{\circledR}$ en el canal medular. De esta forma, el espacio entre el clavo y el endostio fue ocupado por el coágulo, que se formó normalmente.

En el pos-operatorio, los animales recibieron morfina $3 \mathrm{mg} / \mathrm{kg} / 12 \mathrm{~h} / 2$ días/sc y $40.000 U \mathrm{UI} / \mathrm{kg} / 24 \mathrm{~h} / 2$ días/im de penicilina sódica y procaínica y las heridas fueron curadas por diez días.

Prueba mecánica de tracción. Para los estudios mecánicos a los 8, 30, 60, 90 y 120 días posteriores a las cirugías, fue necesario la eutanasia de cuatro conejos por fecha de evaluación con sobredosis de tiopental sódico, por vía intravenosa, previa tranquilización con levopromacina por vía intramuscular.

Las tibias de los animales fueron mantenidas inmersas en solución salina a $0,9 \%$, a temperatura constante de $37^{\circ} \mathrm{C}$ (Ono et al. 2000; Perez E Ortega 2005). Estas fueron seccionadas con cierra manual eléctrica, buscando la remoción de la epífisis proximal, con el propósito de exponer $1 \mathrm{~cm}$ del clavo intramedular, de tal forma que el conjunto tibia-clavo tuviera $7 \mathrm{~cm}$ de longitud, con espesura propia de la tibia. Para acoplamiento de la muestra al mordiente inferior de la Máquina Universal de test de tracción fue necesario hacer un molde, utilizándose tubo de PVC de $6,25 \mathrm{~cm}$ de diámetro y $6 \mathrm{~cm}$ de largo perforado en la mitad, para que permitiera la introducción de la epífisis distal de la tibia, que fue, entonces, fijada con resina acrílica activada químicamente (metilmetacrilato).

Los cuerpos de prueba fueron colocados verticalmente en la máquina de ensayo universal, de tal forma que $1 \mathrm{~cm}$ del clavo intramedular permaneciera fijo al mordiente superior y el dispositivo de PVC fuese adaptado al mordiente inferior, consiguiendo así el encaje de cada muestra (Figura 1). Enseguida, cada uno de los cuerpos de prueba, tratado y control, se sometieron al test de tracción a una fuerza inicial de aproximadamente $25 \mathrm{~g}$ y una velocidad constante de un $\mathrm{mm} /$ minuto, hasta la soltura del clavo.

Los resultados de la prueba mecánica de tracción fueron interpretados comparando cada fecha de sacrificio entre los grupos tratados y control, con análisis estadístico realizado por el test de Friedman, significancia fijada en $p<0,05$. El comportamiento de las medias para ambos grupos, se representaron por medio de una línea de tendencias.

\section{RESULTADOS Y DISCUSIÓN}

La prueba mecánica de tracción puede ser expresado en Newton o en kilogramos fuerza $(\mathrm{kg} \mathrm{F})$ y registra la fuerza máxima que se necesita para apartar un cuerpo de su centro de gravedad, siendo utilizado en el presente trabajo, para determinar la resistencia máxima ofrecida por la interfase hueso-clavo intramedular, a través del compuesto de hidroxiapatita - lignina. 


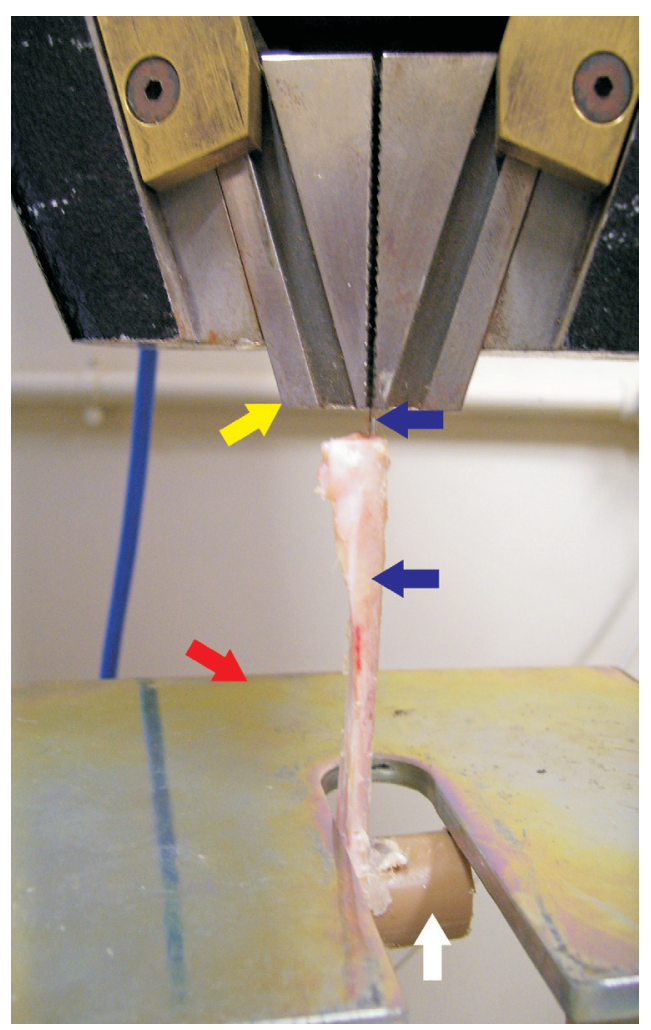

Figura 1. Fotografía del acoplamiento de la muestra tibia-clavo intramedular de conejo (flecha azul) en el mordiente superior (flecha amarilla) y en el mordiente inferior (flecha roja) de la máquina universal para realización de la prueba mecánica de tracción. Molde de PVC (flecha blanca).

En cada grupo el comportamiento de las medias de la prueba mecánica de tracción fue linear creciente, observándose una diferencia cuantitativa considerable al comparar las últimas tres fechas de evaluación con las dos primeras, siendo aun mayor al comparar la evaluación final con la evaluación inicial (Tabla 1).

Al comparar las medias del grupo tratado con las del grupo control, se puede afirmar que las medias del tratado fueron mayores que aquellas del control en cada uno de los días evaluados y la máxima diferencia ocurrió a los 120 días de la evaluación, donde el grupo experimental tratado mostró el doble de la media del grupo control (Tabla 1). Este hecho, posiblemente, estuvo influenciado por las características del compuesto de hidroxiapatita - lignina utilizado, donde el tamaño de sus poros, su extensión y la inter-conectividad entre ellos, permitió la óseo-integración, llevando a una mayor resistencia en la fijación del conjunto implante - hueso (Pollick et al. 1995; Pommer et al. 1997; Mangano et al. 2006). A pesar que en este trabajo no se utilizaron clavos metálicos revestidos industrialmente

Tabla 1. Medias de la prueba mecánica de tracción (kg F) implantado en la tibia de conejos tratada con hidroxiapatita - lignina (GT) y en la tibia sin tratamiento con hidroxiapatita - lignina (GC) durante los días de evaluación.

\begin{tabular}{|c|c|c|c|c|c|}
\hline \multirow{2}{*}{ Grupos } & \multicolumn{5}{|c|}{ Días posteriores a las cirugias } \\
\cline { 2 - 6 } & 8 & 30 & 60 & 90 & 120 \\
\hline G.T. & 0,11 & 3,51 & 11,00 & 16,40 & 22,25 \\
\hline G.C. & 0,10 & 1,76 & 8,75 & 7,75 & 10 \\
\hline
\end{tabular}


con hidroxiapatita, los resultados obtenidos fueron semejantes a los observados por Sakano et al. (2001), en investigaciones realizadas en humanos utilizando clavos revestidos con hidroxiapatita. En el mismo sentido anterior, los resultados también fueron semejantes a los obtenidos por Sasaki et al. (2000), Tisdel et al. (1994), Vidigal \& Goisman (1997), Vital et al. (2006), quienes afirmaron que los implantes revestidos con hidroxiapatita, comparados con otros de igual diseño, mas no revestidos, en diferentes especies de estudio, presentaron una mayor fuerza de fijación.

Al analizar estadísticamente el grupo tratado con el grupo control y entre días de un mismo grupo, no se observaron diferencias, de acuerdo con el test de Friedman y Duun's, respectivamente (Figura 2).

El comportamiento de las medias del grupo tratado con el compuesto de hidroxiapatita - lignina puede ser explicado por la siguiente ecuación exponencial: $\mathrm{y}=0,0041 \mathrm{X}^{1,7837}$, donde $\mathrm{y}=$ media del test mecánico de tracción en kg $F$ para el grupo tratado con el compuesto $\mathrm{y}, \mathrm{x}=$ unidades en días. El coeficiente de determinación explica en $88 \%$ el comportamiento de las medias del grupo tratado con el compuesto en el período del estudio.

El comportamiento de las medias del grupo control puede ser explicado por la siguiente ecuación exponencial: $\mathrm{y}=0,0036 \mathrm{X}^{1,7491}$, donde $\mathrm{x}=$ unidades en días. El coeficiente de determinación explica en $94 \%$ el comportamiento de las medias del grupo control en el período del estudio.

A pesar de observarse diferencias cuantitativas con la evolución del tiempo en cada día de observación entre los grupos tratados y control y entre los días de un mismo grupo, el análisis estadístico no evidenciÓ diferencias significativas, de acuerdo con el valor de "p".

En un estudio preliminar realizado recientemente por Martínez (2007), (Profesor de Cirugía animal. FMVZUniversidad de Córdoba-Colombia, comunicación personal), los resultados demostraron que la fuerza necesaria para fracturar la cortical tibial de conejo por la técnica de tracción mecánica, fue de 92,33kg. Basado

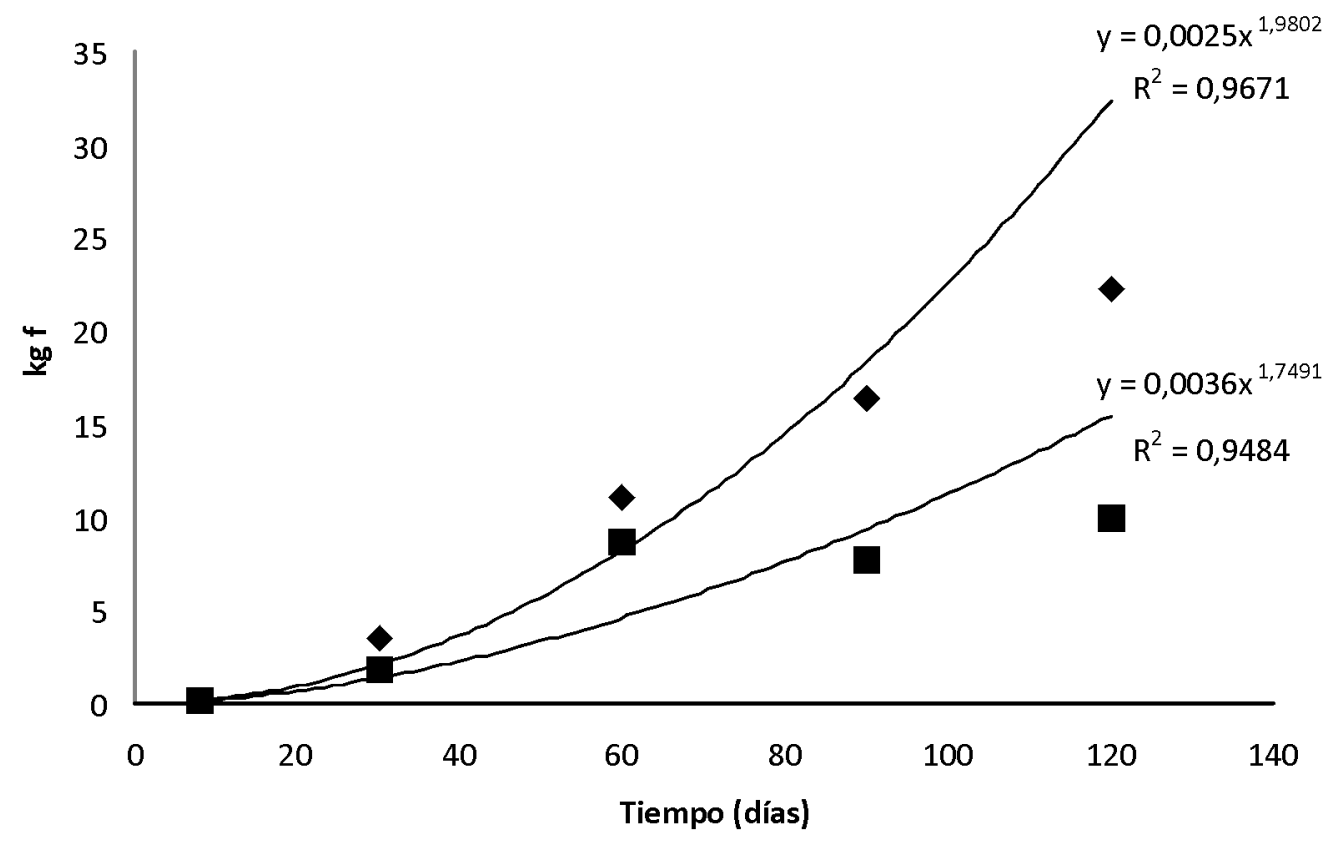

- GT $\square \mathrm{GC} \longrightarrow$ Potencial (GT) $\longrightarrow$ Potencial (GC)

Figura 2. Línea de tendencias de las medias de la prueba mecánica de tracción de los clavos intramedulares en las tibias de conejos del grupo tratado (GT) con hidroxiapatita - lignina y del grupo control (GC) en los días del estudio experimental. 
en el dato anterior, los clavos intramedulares del grupo tratado opusieron una resistencia equivalente al $24 \%$ de la fuerza máxima, media que se necesitó para fracturar la tibia de conejo y que para el grupo control esta fuerza correspondió a $10 \%$.

AGRADECIMIENTOS: Los autores agradecen al laboratorio JHS por proporcionar el compuesto experimentado, a la Universidad de Córdoba - Colombia y a la Universidad Federal de Viçosa - Brasil. Conflicto de interés: El manuscrito fue preparado y revisado con la participación de todos los autores, quienes declaramos que no existe ningún conflicto de intereses que ponga en riesgo la validez de los resultados presentados. Financiación: El presente estudio fue financiado por el Laboratorio JHS-Sobará-Brasil, la Universidad de Córdoba - Colombia y a la Universidad Federal de Viçosa - Brasil.

\section{BIBLIOGRAFÍA}

1. AYERS, R.; SIMSKE, S.; NUNES, C.; WOLFORD, L. 1998. Longterm bone ingrowth an Residual microhardness of porous block hydroxyapatite implants in humans. J. Oral Maxilofacial Surg. (EEUU) 56(6):1297-1301.

2. BORGES, A.; REZENDE, C.; RIBEIRO, M.; MELO, E.; NÒBREGA, P. 2000. Hidroxiapatita sintética como substituto ósseo em defeito experimental provocado no terço proximal da tíbia em cães: aspectos à microscopia eletrônica de transmissão. Arq. Bras. Med. Vet. Zootec. 52(6):35-42.

3. BOSTROM, M.P. 1998. Expression of bone morphogenetic proteins in fracture healing. J. Clin. Orthop. (EEUU) 335:116-123.

4. FEHLBERG, A.F. 2001. Hidroxiapatita sintética pura (hap-91), hidroxiapatita sintética associada ao colágeno (col.hap-91) e hidroxiapatita sintética associada ao lipossoma (int.hap-91) como substitutos ósseos em defeitos provocados na tíbia de cães: aspectos da osteointegração à microscopia de luz transmitida. hidroxiapatita sintética pura (hap-91), hidroxiapatita sintética associada ao colágeno (col.hap-91) e hidroxiapatita sintética associada ao lipossoma (int.hap-91) como substitutos ósseos em defeitos provocados na tíbia de cães: aspectos da osteointegração. Arq. Bras. Med. Vet. Zootec. 53(4):431-436.

5. FRANCO, K.L.; BORGES, A.P.B.; VILÓRIA, M.I.V. 2001. Hidroxiapatita sintética pura, hidroxiapatita associada ao colágeno e hidroxiapatita sintética associada ao lipossoma como substitutos ósseos em defeitos provocados na tíbia de cães: aspectos da osteointegração à microscopia de luz transmitida. Arq. Bras. Med.Vet. Zootec. 53:431436.

6. HEMMERLÉ, J.; CUISINIER, G.; SCHULTZ, P., VOEGEL, C. HRTEM. 1997. Study of biological crystal growth mechanisms in the vicinity of implanted synthetic hydroxyapatite crystals. J. Dental Res. (EEUU). 76(2):682-687.

7. LEGEROS, R.Z. 2002. Properties of osteoconductive biomaterials: calcium phosphates. Clin. Orthop. Rel. Res. (EEUU). 395:81-98.

8. LIZARBE, M.A. 2007. Sustitutivos de tejidos: de los biomateriales a la ingeniería tisular. Rev. Real Acad. Cienc. Exactas, Físicas y Naturales (España). 101(1):227-249.

9. MANGANO, C.; SCARANO, A.; IEZZI, G.; ORSINI, G.; PERROTTI, V.; MANGANO, F.; MONTINI, S.; PICCIRILLI, M.; PIATELLI, A. 2006. Maxillary sinus augmentation using an engineered porous hydroxyapatite: a clinical, histological, and transmission electron microscopy study in man. J. Oral Implantology. 32(3):122-131.

10. MIRANDA, A.L.; FRANCO DA SILVA, L.A.; TAVARES, G.A.; COUTO, D.O.; ANDRÉIA, V.A.; GUIMARÃES DE MIRANDA, H. 2006. Abraçadeira de náilon: resistência à tração em testes físicos e seu emprego como cerclagem no fêmur de cães. Rev. Ciência Animal Brás. 7(3):299-307.

11. MONCHAU, F.; LEFÈVRE, A; DESCAMPS M. 2002. In Vitro studies of human and rat osteoclast activity on tricalcium phosphate, calcium carbonate. $\mathrm{J}$ Biomolecular Eng. 19(269):143-152. 
12. MORONI, A; CAJA, V.L., EGGAR, E. GOTTSAUNERWOLF, F. TRINCHESE, L.; ROLLO, G.; CHAO, E.Y. 1996. Porous titanium implants with and without hydroxiapatite coating. En: Ravaglioli, A.; Krajewsky, A. (Eds.) Bioceramics and the human body. Barking: Elsevier Science Publishers, Ltd. 4:335-342.

13. MORONI, A.; ASPENBERG, P.; TOKSVIG, L.; FALZARANO, G.; GIANNINI, S. 1998a. Enhanced fixation with hyd. Coated pins. Clin. Orthop. Rel. Res. (EEUU). 346:171-177.

14. MORONI, A.; TOKSVIG, L.S.; MALTERELLO, M.C.; ORIENTI, L.; STEA, S.; GIANNINI, S. 1998b. Acomparison of hyd-coated, titanium-coated and uncoated tapered external-fixation pins. J. Bone Joint Surg. Am. (EEUU) 80:547-554.

15. ONO, I.; TATESHITA, T.; NAKAJIMA, T. 2000. Evaluation of a high density polyethylene fixing system for hydroxyapatite ceramic implants. J. Biomaterials (EEUU). 21:143-151.

16. PÉREZ, Á.; ORTEGA, V.; MESEGUER L.; ALCARAZ M.; SEPULVEDA P.; CLAVEL-NOLLA, M. 2005. Implante óseo de la espuma de Hidroxi- apatita-09. Estudio experimental en conejos. Esp. Patología. 38(1):14-20.

17. PIERMATTEI, D.L.; FLO, G.L.; DECAMPO, C.E. 2006. Handbook of SmallAnimal Orthopedics and Fracture Repair. Ed Missour: Saunders Elsevier (EEUU). p.139-140.

18. POLLICK, S.; SHORS, E.C.; HOLMES, R.E.; 1995. Bone formation and implant degradation of coraline porous ceramics placed in bone and ectopic sites. J. Oral Maxillfac. Surg. (EEUU). 53:915-922.
19. SAKANO, H.; KOSHINO, T.; TAKEUCHI, R. 2001. Treatment of the instable distal radius fracture with external fixation and a hydroxyapatite spacer. J. Hand Surg. (EEUU). 26:923-929.

20. SASAKI, S.U.; STUGINSKI, R.M.; MATTAR, Jr. R. 2000. Estudo biomecânico comparativo da resistência à tração entre dois tipos diferentes de miniâncoras de sutura. Rev. Bras. Ortop. 35:231234.

21. SINGH, R.; DAHOTRE, N.B. 2007. Corrosion degradation and prevention by surface modification of biometallic materials. J. Mater Sci. Mater Med. (EEUU). 18:725-751.

22. TISDEL, C.L.; GOLBERG, V.M.; PARR, J.A.; BENSUSAN, J.S.; STAIKOFF, L.S.; STEVENSON, S. 1994. The influence of hydroxyapatite and tricalcium phosphate coating on bone growth into titanium fiber-metal implants. J. Bone Joint Surg. Am. 76:159-171.

23. VIDIGAL Jr., G.M.; GOISMAN, M. 1997. Osseointegração $\times$ biointegração: uma análise crítica. Rev. Bras. Odontol. 4:54.

24. VITAL, C.C.; BORGES, A.P.B.; FONSECA, C.C.; TSIOMIS, A.C.; CARVALHO, T.B.; FONTES, E.B.; SENA, M.P.; FÓFANO, G. 2006. Biocompatibilidade e comportamento de compósitos de hidroxiapatita em falha óssea na ulna de coelhos. Arq. Bras. Med. Vet. Zootec. 58(2):175-183.

Recibido: Marzo 26 de 2009

Aceptado: Septiembre 21 de 2009 